\title{
Synthesis and antitumor activity of novel silibinin and 2,3-dehydrosilybin derivatives with carbamate groups
}

\author{
Qiuchan $\mathrm{Wu}^{1} \cdot$ Jiang Zeng ${ }^{2} \cdot$ Jinfu Dong $\mathbb{1}^{3}$
}

Received: 1 November 2021 / Accepted: 20 January 2022 / Published online: 15 February 2022

(c) The Author(s), under exclusive licence to Springer Science+Business Media, LLC, part of Springer Nature 2022

\begin{abstract}
A novel series of silibinin and 2,3-dehydrosilybin derivatives bearing carbamate groups were designed, synthesized and their in vitro anticancer activities were screened against human cancer cell lines including MCF-7, NCI-H1299, HepG2 and HT29 by CCK-8 assay. The results showed that most of the compounds significantly suppressed the proliferation of tested cancer cells. Among them, compounds $2 \mathbf{h}, \mathbf{3 h}$ and $\mathbf{3 f}$ demonstrated markedly higher antiproliferative activity on MCF-7 cells with $\mathrm{IC}_{50}$ values of $2.08,5.54$ and $6.84 \mu \mathrm{M}$, respectively. Compounds $\mathbf{3 e}, \mathbf{3 g}$ and $\mathbf{2 g}$ displayed better cytotoxic activity against NCI-H1299 cells with $\mathrm{IC}_{50}$ values of $8.07,8.45$ and $9.09 \mu \mathrm{M}$, respectively. Compounds 3g, 3c and $\mathbf{3 h}$ exhibited a promising inhibitory effect against $\mathrm{HepG} 2$ cells with $\mathrm{IC}_{50}$ values of $8.88,9.47$ and $9.99 \mu \mathrm{M}$, respectively. Compounds $3 \mathbf{e}, \mathbf{2 e}$ and $\mathbf{3 c}$ revealed effective biological potency on HT29 cells with IC $_{50}$ values of $6.27,9.13$ and $9.32 \mu \mathrm{M}$, respectively. In addition, the outcomes of the docking studies between compounds 2f, 2h, 3e, 3g and Hsp90 receptor (PDB ID: 4AWO) suggest the possible mechanism of inhibition against MCF-7 cell lines.
\end{abstract}

\section{Graphical abstract}

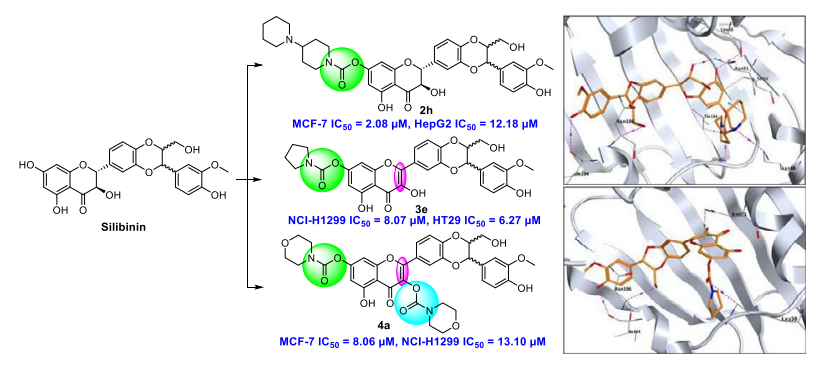

Keywords Silibinin $\cdot 2,3$-dehydrosilybin $\cdot$ Carbamate $\cdot$ Synthesis $\cdot$ Anticancer $\cdot$ Docking

These authors contributed equally: Qiuchan Wu, Jiang Zeng, Jinfu Dong

Supplementary information The online version contains supplementary material available at https://doi.org/10.1007/s00044022-02854-6.

Jinfu Dong

dongjinfu@163.com

1 Department of Hematology and Medical Oncology, Tianjin Fifth Central Hospital, Tianjin 300450, China

\section{Introduction}

Cancer is an abhorrent disease with extremely high mortality, caused by abnormal proliferation of human cells $[1,2]$. It is estimated that there are approximately 19.3 million new cancer cases in 2020 across the world, and

2 Department of Pharmacy, The Fourth Affiliated Hospital of Guangxi Medical University, Liuzhou 545005, China

3 Department of Medicinal Chemistry, Central South University, No. 172 Tongzipo Road, Changsha, Hunan 410013, China 


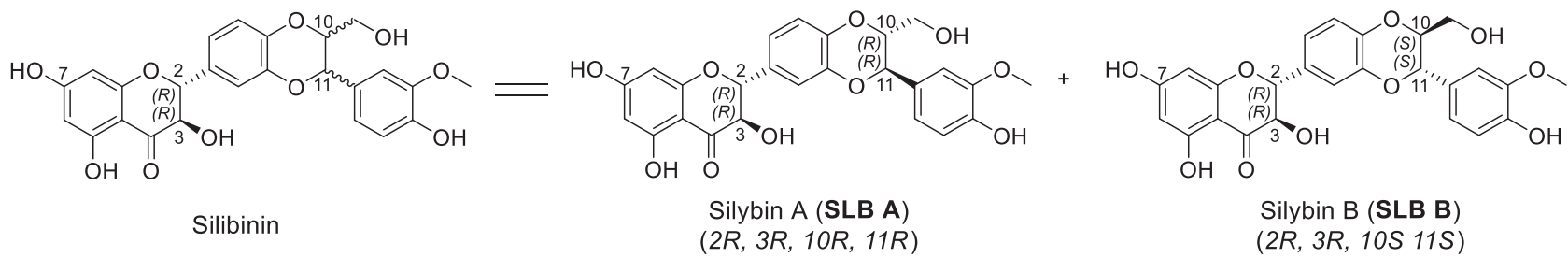<smiles>C#COc1cc([C@H]2Oc3ccc(-c4oc5cc(O)cc(O)c5c(=O)c4O)cc3O[C@H]2CO)ccc1O</smiles>

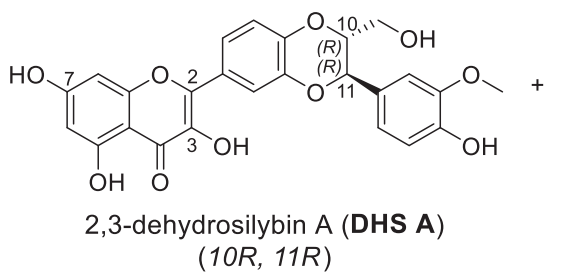<smiles>COc1cc([C@@H]2Oc3ccc(-c4oc5cc(O)cc(O)c5c(=O)c4O)cc3O2)ccc1O</smiles>

2,3-dehydrosilybin B (DHS B) (10S, 11S)

Fig. 1 Chemical structures of silybiin (SLB A, SLB B) and 2,3-dehydrosilybin (DHS A, DHS B)

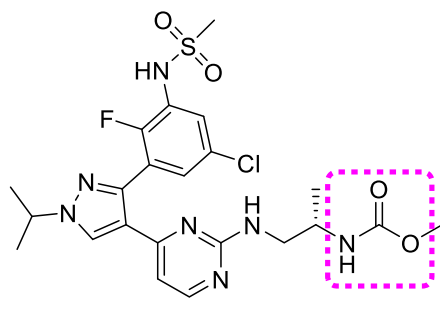

Encorafenib (B-raf inhibitor)

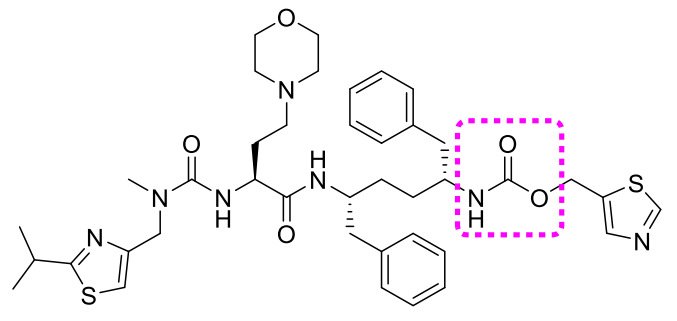

Cobicistat (CYP3A inhibitor)

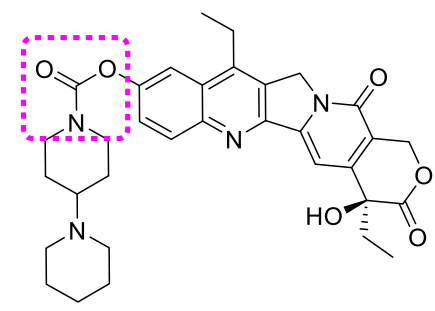

Irinotecan (TOP1 inhibitor)

Fig. 2 Chemical structures of encorafenib, cobicistat and irinotecan

this number will surge remarkably to 28.4 million in 2040 [3]. Natural products have been widely investigated and eventually developed into drugs, especially anticancer chemotherapeutics due to their molecular diversity and novelty $[4,5]$. Silymarin is an active extract isolated from the seeds of the milk thistle plant, Silybum marianum, which contains approximately 65-80\% flavonolignans (silybinin, isosilybin, silychristin, silydianin, 2,3-dehydrosilybin) with small amounts of flavonoids and about 20-30\% of unidentified polymeric phenolic components [6-8]. Silibinin, the main active ingredient of silymarin, is a roughly $1: 1$ mixture of two diastereoisomers named as silybin A (SLB A) and silybin B (SLB B), while the 2,3dehydrosilybin (DHS) consists of a mixture of two enantiomers, namely, DHS A and DHS B [9] (Fig. 1). Silibinin and DHS have been shown to possess anticancerogenic and proapoptotic activity both in vitro and in vivo [10]. Silibinin, as positive control, exhibits anticancer activity with typical $\mathrm{IC}_{50}$ value of around $50-200 \mu \mathrm{M}$ in various cancers $[11,12]$, while at lower concentrations, DHS induces apoptosis at $30-50 \mu \mathrm{M}$ when treated alone [13]. Encouragingly, silybin is currently under phase II clinical trials in the US for prostate cancer treatment [14]. Despite these encouraging results, their use as an antitumor drug is considerably hampered by their small solubility in water which caused by highly hydrophobic characteristic and low acidity [15-19]. To improve the druggability and enhance antiproliferative activity of silibinin and DHS, structural modifications are essential [20, 21].

Organic carbamates are structural motifs of many approved pharmaceutical drugs [22, 23], such as Encorafenib (approved in 2018), Cobicistat (approved in 2013), Irinotecan (approved in 1994), etc (Fig. 2). Structurally, partly owing to the amide-ester hybrid nature, the carbamate functional group displays good chemical and proteolytic stabilities [24]. Furthermore, carbamates have been demonstrated to have the ability to promote interand intramolecular interactions with the biological targets. In addition, numerous studies have indicated that structural modifications by introducing carbamate groups greatly improve the water solubility and biological activity of the drugs [25-30]. Based on the above information, a class of silibinin and 2,3-DHS derivatives bearing carbamate groups were designed and synthesized. Their antiproliferative activities were investigated and the structure-activity relationships (SARs) were discussed. 
<smiles>[R]C(=O)Oc1cc(O)c2c(c1)O[C@H](c1ccc3c(c1)O[C@H](c1ccc(O)c(OC)c1)[C@H](CO)O3)[C@H](O)C2=O</smiles>

Scheme 1 Synthesis of carbamate-containing silibinin and 2,3-dehydrosilybin derivatives

\section{Results and discussion}

\section{Synthesis of silibinin and 2,3-dehydrosilybin derivatives}

Scheme 1 presents the protocol for the synthesis of carbamatecontaining silibinin and 2,3-DHS derivatives. The reaction of silybin and carbamyl chloride 1a-i in the presence of $\mathrm{N}, \mathrm{N}$ diisopropylethylamine (DIPEA), 4-Dimethylaminopyridine (DMAP) and tetrahydrofuran (THF) as the solvent produced the desired product $\mathbf{2 a - i}$ in yields of $10.8-52.8 \%$. When the reaction base and the solvent were replaced by potassium carbonate $\left(\mathrm{K}_{2} \mathrm{CO}_{3}\right)$ and $N, N$-dimethylformamide (DMF), mono-substituted 2,3-DHS derivatives 3a-i were produced in yields of 11.2-39.9\%. Surprisingly, disubstituted 2,3-DHS derivatives $\mathbf{4 a}$ and $\mathbf{4 b}$ were also synthesized selectively with yields of 11.1 and $27.7 \%$, respectively, by simply increasing the amount of $\mathrm{K}_{2} \mathrm{CO}_{3}$ and carbamyl chloride 1a-b. Twenty novel silibinin and 2,3-DHS derivatives bearing carbamate groups were synthesized, and their structures were characterized by ${ }^{1} \mathrm{H}$ NMR, ${ }^{13} \mathrm{C}$ NMR and mass spectroscopy. As expected, it was found that all target compounds exhibited substantially higher aqueous solubility compared with untreated silibinin and DHS.

\section{In vitro cytotoxicity screening of the synthesized compounds}

The in vitro cytotoxicity of the synthesized target compounds (2a-i, 3a-i and $\mathbf{4 a - b}$ ) against four human cancer cell lines including MCF-7 (breast carcinoma), NCI-H1299 (lung carcinoma), HepG2 (liver carcinoma) and HT29 (colon carcinoma) was assessed by cell counting kit-8 (CCK-8) assay. The $\mathrm{IC}_{50}$ values of the compounds against the tested cell lines are summarized in Table 1. Silibinin and 2,3-DHS were used as control, which exhibited poor antiproliferative activity against all the four types of cancer cells with almost all $\mathrm{IC}_{50}$ values $>20 \mu \mathrm{M}$. As expected, compared with positive drugs, most of their derivatives bearing carbamate groups exhibited significantly enhanced antitumor activity against the tested tumour cell lines. The majority of the compounds demonstrated antitumor activity with $\mathrm{IC}_{50}$ values at low micromolar concentrations. In particular, a series of derivatives exhibited potency in the single-digit micromolar range, such as compounds $2 \mathbf{h}, 3 \mathbf{h}, \mathbf{3 f}, \mathbf{3 g}, \mathbf{4 b}, \mathbf{4 a}$ and $\mathbf{2 g}$ showed better cytotoxic activity against MCF-7 cells with $\mathrm{IC}_{50}$ values of $2.08,5.54,6.84,7.96,8.05,8.06$ and $8.24 \mu \mathrm{M}$, compounds $\mathbf{3 e}, \mathbf{3 g}$ and $\mathbf{2 g}$ displayed high antiproliferative activity against NCI-H1299 cells with $\mathrm{IC}_{50}$ values of $8.07,8.45$ and $9.09 \mu \mathrm{M}$, compounds $\mathbf{3 g}, \mathbf{3 c}$ and $\mathbf{3 h}$ revealed effective biological potency on HepG2 cells with $\mathrm{IC}_{50}$ values of $8.88,9.47$ and $9.99 \mu \mathrm{M}$, compounds $\mathbf{3 e}, \mathbf{2 e}$, $\mathbf{3 c}$ and $\mathbf{2 f}$ exhibited a promising inhibitory effect against HT29 cells with $\mathrm{IC}_{50}$ values of $6.27,9.13,9.32$ and $9.86 \mu \mathrm{M}$, respectively. Among the twenty screened compounds, seven compounds (2f, $2 \mathrm{~g}, 3 \mathbf{3 b}, \mathbf{3 e}, \mathbf{3 g}, \mathbf{4 a}$ and $\mathbf{4 b}$ ) have exhibited superior potency in anticancer activity on all tested cell lines. Especially compound $\mathbf{2 g}$ (MCF-7 $\mathrm{IC}_{50}=$ $8.24 \mu \mathrm{M}$, NCI-H1299 $\mathrm{IC}_{50}=9.09 \mu \mathrm{M}$, HepG2 $\mathrm{IC}_{50}=$ $\left.13.96 \mu \mathrm{M}, \quad \mathrm{HT} 29 \mathrm{IC}_{50}=10.80 \mu \mathrm{M}\right)$ and 3g (MCF-7 
Table 1 In vitro antiproliferative activity of silibinin analogues $\mathbf{2 a - i}$, 2,3-dehydrosilybin derivatives $\mathbf{3 a - i}$ and $\mathbf{4 a - b}$

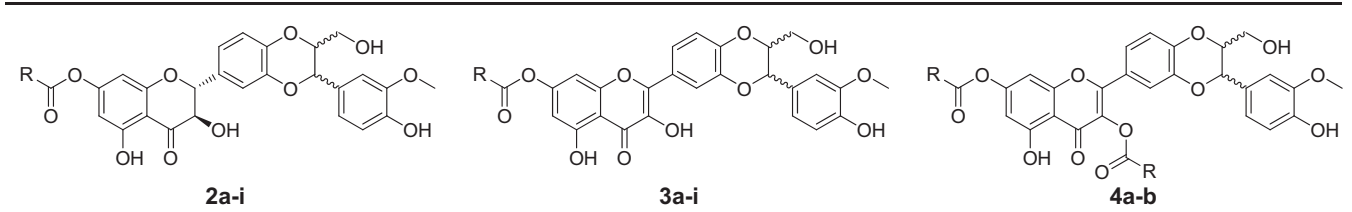

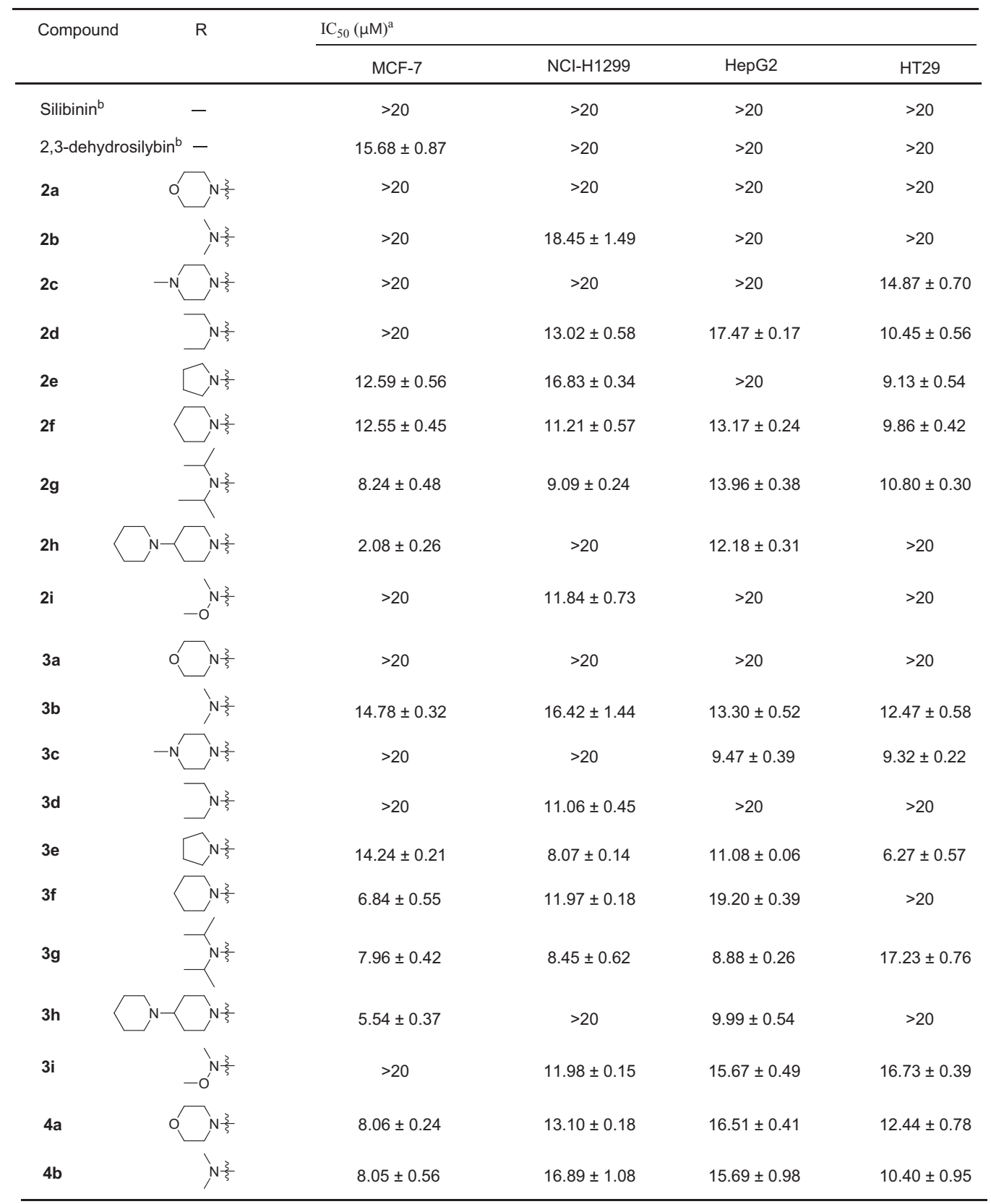

${ }^{\mathrm{a}}$ Values expressed are means \pm SEM of three parallel measurements

${ }^{\mathrm{b}}$ Reference compound

$\mathrm{IC}_{50}=7.96 \mu \mathrm{M}$, NCI-H1299 $\mathrm{IC}_{50}=8.45 \mu \mathrm{M}$, HepG2 $\mathrm{IC}_{50}$ $=8.88 \mu \mathrm{M}$, HT29 $\left.\mathrm{IC}_{50}=17.23 \mu \mathrm{M}\right)$ have demonstrated stronger broad-spectrum antitumor activities.
Substituent $\mathrm{R}$ plays a crucial role in the biological activity of this group of drugs. According to the structureactivity relationship study, the $N, N$-dimethylcarbamate 

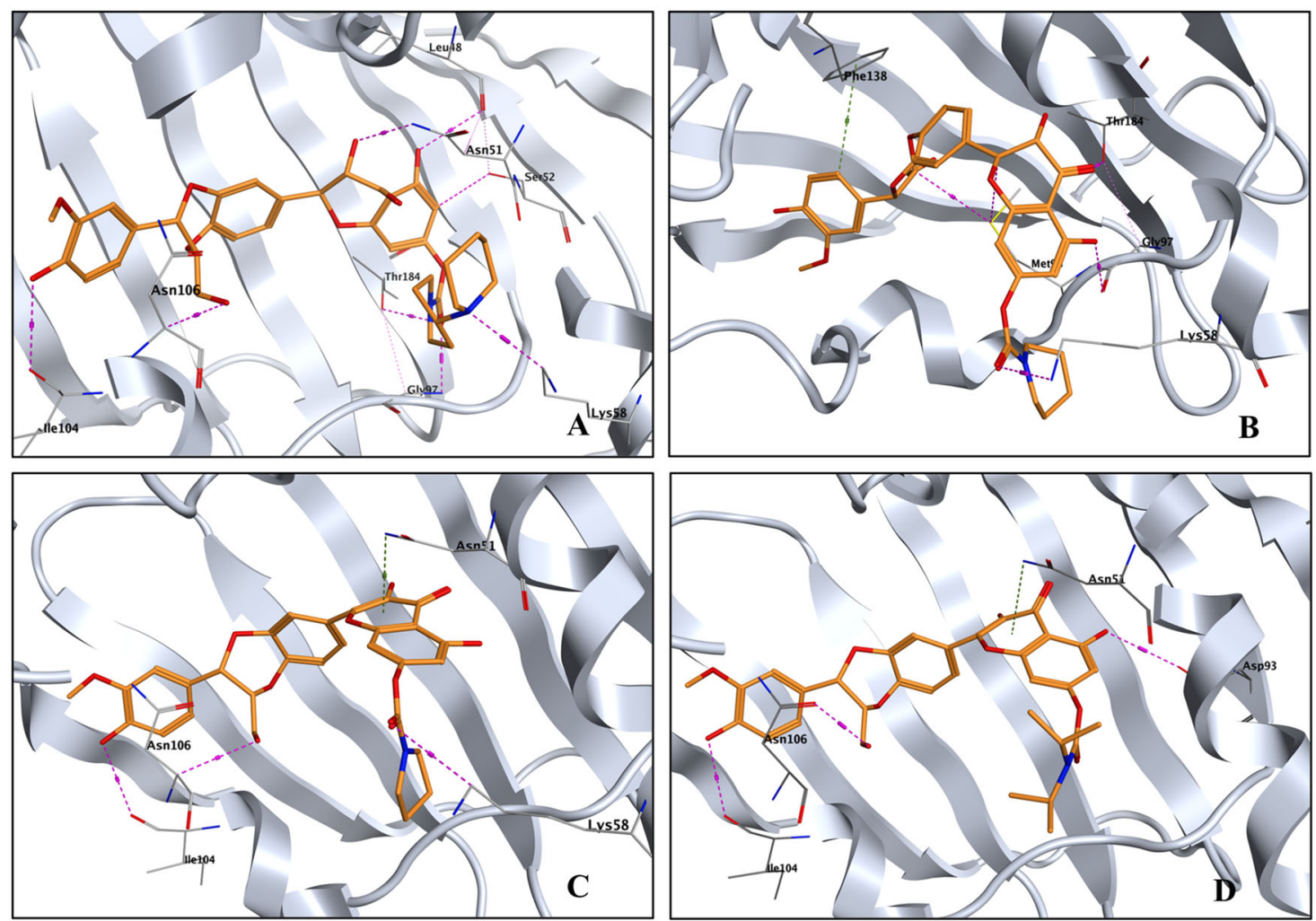

Fig. 3 Docked positions of compound $2 \mathbf{h}(\mathbf{A}), \mathbf{2 f}(\mathbf{B}), \mathbf{3 e}(\mathbf{C})$ and $\mathbf{3 g}(\mathbf{D})$ inside active site of Hsp90 (PDB ID: 4AWO)

derivative $2 \mathbf{b}(18.45 \mu \mathrm{M})$, the $N, N$-diethyl carbamate analogue $2 \mathbf{d}(13.02 \mu \mathrm{M})$, and the $N, N$-diisopropylcarbamate substitute $2 \mathrm{~g}(9.09 \mu \mathrm{M})$ showed a trend toward more potent anticancer activity against NCI-H1299 cells with increasing lipophilicity of the carbamate residues in the molecule. The same tendency in cyclic carbamate derivatives was also observed, with the pyrrolidine-1-carboxylate derivative $\mathbf{3 e}$ $(14.24 \mu \mathrm{M})$, the piperidine-1-carboxylate analogue $\mathbf{3 f}$ $(6.84 \mu \mathrm{M})$, and the $\left[1,4^{\prime}\right.$-bipiperidine $]-1^{\prime}$-carboxylate substitute 3h $(5.54 \mu \mathrm{M})$ showed increasing cytotoxic activity against MCF-7 cells, indicating positive effect of the lipophilic substituents on the activity of these compounds. The derivatives bearing morpholine-4-carboxylate group 2a and 3a generally exhibited much higher $\mathrm{IC}_{50}$ values (all $>20 \mu \mathrm{M}$ ) comparing to compounds bearing the piperidine-1carboxylate group $\mathbf{2 f}$ and $\mathbf{3 f}$, which indicated that the additional oxygen atom in the morpholino group interferes greatly with the antiproliferative activities. More interestingly, $\mathrm{C} 3-\mathrm{OH}$ and $\mathrm{C} 7-\mathrm{OH}$ disubstituted derivative of 2,3DHS 4a (MCF-7 $\mathrm{IC}_{50}=8.06 \mu \mathrm{M}$, NCI-H1299 $\mathrm{IC}_{50}=$ $13.10 \mu \mathrm{M}, \quad$ HepG2 $\mathrm{IC}_{50}=16.51 \mu \mathrm{M}, \quad \mathrm{HT} 29 \quad \mathrm{IC}_{50}=$ $12.44 \mu \mathrm{M})$ exhibited significantly stronger inhibition potency than $\mathrm{C} 7-\mathrm{OH}$ mono-substituted analogue 3a (all $\mathrm{IC}_{50} \mathrm{~S}>20 \mu \mathrm{M}$ ), which suggested that the double simultaneous substitution strategy is a promising approach in improving anticancer activity. Moreover, it was observed that silibinin derivatives $\mathbf{2 a}-\mathbf{2 h}$ had similar activity with the corresponding analogues of 2,3-DHS $\mathbf{3 a -} \mathbf{3} \mathbf{h}$ against NCIH1299 cells, which suggested that dehydrogenation of silibinin derivatives at both 2- and 3-positions $(\mathrm{CHCH} \rightarrow \mathrm{C}=\mathrm{C})$ had limited the influences on the antiproliferative activities, especially toward target NCIH1299 cells.

\section{Molecular docking studies}

Regarding to the molecular mechanisms of silibinininduced apoptosis in breast cancer (MCF-7), numerous studies have reported multiple biological targets, such as Hsp90 [31, 32], BCL-2 [33], ERK [34], Akt [34], COX-2 [35], MMP-9 [35, 36], etc. The docking studies of the silibinin and 2,3-DHS analogues (2f, $\mathbf{2 h}, \mathbf{3 e}$ and $\mathbf{3 g}$ ) were conducted against six protein kinases to investigate the putative mechanism of actions of these novel compounds by MOE. The outcomes of the docking studies showed comparatively higher docking scores against the Hsp90 (4AWO) so that it was chosen for virtual screening study. The results indicated that, $\mathbf{2 h}$ bound strongly to Hsp90 receptor with minimum binding energy $-9.88 \mathrm{kcal} / \mathrm{mol}$, while the binding energy of $\mathbf{2 f}, \mathbf{3 e}$ and $\mathbf{3 g}$ are $-8.91,-8.39$ and $-9.11 \mathrm{kcal} / \mathrm{mol}$, respectively.

As illustrated in Fig. 3, docking results revealed that the silibinin analogue $\mathbf{2 h}$ had eight $\mathrm{H}$-bond interactions with the residues Ser52, Leu48, Ile104, Asn51, Asn106, Gly97, 
Thr184 and Lys58 at distances of $3.39,3.05,3.32,2.68$, $3.24,2.82,2.90$ and $3.34 \AA$ in the ligand-binding pocket, respectively. Similarly, 2f-Hsp90 cluster showed six H-bonds with Met98, Gly97, Met98, Thr184, Lys58 and Phe138 amino acids at distances of 4.16 (12CH $\cdots \mathrm{S}$ bond), 2.87, 3.88 (33 $\mathrm{CH} \cdots \mathrm{S}$ bond), 3.21, 3.13 and $4.62 \AA$, respectively. Four major H-bonds were formed on Ile104, Asn106, Lys58 and Asn51 amino acid residues for compound 3e with distances of $3.29,3.25,3.41$ and $3.93 \AA$, respectively. In addition, $\mathbf{3 g}$ established four H-bonds with Asp93, Asn106, Ile104 and Asn51 amino acids with the corresponding distances being 3.33, 2.82, 3.31 and $3.85 \AA$, respectively. The docking results are in good agreement with in vitro experimental $\mathrm{IC}_{50}$ values against MCF-7 cells shown in Table 1.

\section{Conclusions}

Modified on the basis of the silibinin and 2,3-DHS scaffold, 20 novel carbamate derivatives were successfully designed, synthesized, and evaluated against four human cancer cell lines including MCF-7, NCI-H1299, HepG2 and HT29 by employing CCK-8 method. Importantly, compounds $\mathbf{2 h}, \mathbf{3 g}$, 3c and 3e displayed as low as single-digit micromolar activity against different cancer cell lines. Molecular docking between compound $\mathbf{2 f}, \mathbf{2 h}, \mathbf{3 e}, \mathbf{3 g}$ and Hsp90 were in good agreement with in vitro experimental $\mathrm{IC}_{50}$ values against MCF-7 cell lines, which disclosed the possible mechanisms of anticancer action. The above findings provided a good starting point for the rational design and development of novel anticancer drugs and Hsp90 inhibitors with silibinin and 2,3-DHS scaffold.

\section{Experimental}

All chemicals and reagents were obtained from Chemical Book, Sigma-Aldrich and were used without further purification. Reactions were monitored by TLC, performed on silica gel glass plates containing GF-254, and visualization on TLC was achieved by UV light. ${ }^{1} \mathrm{H}$ and ${ }^{13} \mathrm{C}$ NMR spectra were recorded on a Bruker DRX 400 NMR instrument. Chemical shifts $(\delta)$ are reported in ppm downfield from internal TMS standard. ESI spectra were recorded on a Qstar mass spectrometer. Melting points were determined with an electro thermal melting point apparatus, and are uncorrected.

\section{General procedure for the synthesis of compounds 2a-i}

To a solution of silibinin $(241.2 \mathrm{mg}, 0.50 \mathrm{mmol}, 1.0 \mathrm{eq})$ in THF $(3.6 \mathrm{~mL}, 15.0 \mathrm{vol})$ was added DIPEA $(129.2 \mathrm{mg}$,
$1.0 \mathrm{mmol}, 2.0 \mathrm{eq}), 4-\mathrm{DMAP}(12.2 \mathrm{mg}, 0.10 \mathrm{mmol}, 0.2 \mathrm{eq})$ and carbamyl chloride 1a-i $(0.53 \mathrm{mmol}, 1.05 \mathrm{eq})$. The mixture was stirred at $25{ }^{\circ} \mathrm{C}$ under $\mathrm{N}_{2}$ atmosphere for $15 \mathrm{~h}$. After completion of the reaction, the reaction mixture diluted with water $(20 \mathrm{~mL})$ and extracted with ethyl acetate $(20 \mathrm{~mL})$ twice. The combined organic layer was washed with brine $(20 \mathrm{~mL})$, dried over $\mathrm{Na}_{2} \mathrm{SO}_{4}$ and filtered. The filtrate was concentrated to give the crude product. The residue was purified by column chromatography $\left(\mathrm{CH}_{2} \mathrm{Cl}_{2} / \mathrm{MeOH}=15: 1\right)$ to give the title compound $\mathbf{2 a - i}$.

(2R,3R)-3,5-dihydroxy-2-(3-(4-hydroxy-3-methoxyphenyl)-2(hydroxymethyl)-2,3-dihydrobenzo[b][1,4]dioxin-6-yl)-4oxochroman-7-yl morpholine-4-carboxylate (2a)

White solid (157.2 mg, purity $96.11 \%$, yield $52.8 \%$ ); mp $148-151^{\circ} \mathrm{C} ;{ }^{1} \mathrm{H}$ NMR (DMSO- $\left.d_{6}, 400 \mathrm{MHz}\right) \delta 11.69$ (s, $1 \mathrm{H}), 9.16(\mathrm{~s}, 1 \mathrm{H}), 7.12(\mathrm{~d}, J=2.08,1 \mathrm{H}), 7.05(\mathrm{dd}, J=9.96$, $1.52,1 \mathrm{H}), 7.03(\mathrm{~d}, J=1.52,1 \mathrm{H}), 7.00(\mathrm{~d}, J=8.28,1 \mathrm{H})$, $6.88(\mathrm{dd}, J=8.23,1.52,1 \mathrm{H}), 6.81(\mathrm{~d}, J=8.08,1 \mathrm{H}), 6.40$ $(\mathrm{d}, J=2.08,1 \mathrm{H}), 6.35(\mathrm{~d}, J=2.32,1 \mathrm{H}), 5.97(\mathrm{~d}, J=6.28$, $1 \mathrm{H}), 5.24(\mathrm{~d}, J=11.56,1 \mathrm{H}), 4.97(\mathrm{br}, 1 \mathrm{H}), 4.93(\mathrm{~d}, J=$ 7.92, 1H), 4.80-4.74 (m, 1H), 4.20-4.17 (m, 1H), 3.78 (s, $3 \mathrm{H}), 3.64-3.62(\mathrm{t}, J=5.04 \mathrm{~Hz}, 4 \mathrm{H}), 3.53(\mathrm{br}, 3 \mathrm{H}), 3.41(\mathrm{br}$, $2 \mathrm{H}), 3.34(\mathrm{~m}, 1 \mathrm{H}) ;{ }^{13} \mathrm{C}$ NMR (DMSO- $\left.d_{6}, 126 \mathrm{MHz}\right) \delta$ $199.9,162.3,162.1,159.2,152.0,148.0,147.4,144.2$, $143.7,130.1,127.8,121.8,120.9,117.1,116.8,115.7$, 112.0, 104.7, 103.0, 101.8, 83.1, 78.5, 76.3, 72.1, 66.1, 60.6, 56.0, 45.1, 44.3; $\mathrm{MS}\left(\mathrm{ESI}^{-}\right) \mathrm{m} / \mathrm{z}$ calcd for $\mathrm{C}_{30} \mathrm{H}_{28} \mathrm{NO}_{12}$ 594.16, found $594.3(\mathrm{M}-\mathrm{H})^{-}$.

(2R,3R)-3,5-dihydroxy-2-(3-(4-hydroxy-3-methoxyphenyl)-2(hydroxymethyl)-2,3-dihydrobenzo[b][1,4]dioxin-6-yl)-4oxochroman-7-yl dimethylcarbamate (2b)

White solid (126.1 mg, purity $98.58 \%$, yield $45.6 \%$ ); $\mathrm{mp}$ $138-141{ }^{\circ} \mathrm{C}$; ${ }^{1} \mathrm{H}$ NMR (DMSO- $\left.d_{6}, 400 \mathrm{MHz}\right) \delta 11.68(\mathrm{~s}$, $1 \mathrm{H}), 9.16(\mathrm{~s}, 1 \mathrm{H}), 7.11(\mathrm{t}, J=1.96,1 \mathrm{H}), 7.04(\mathrm{~d}, 1 \mathrm{H}), 7.02$ (s, 1H), $6.99(\mathrm{~d}, J=8.20,1 \mathrm{H}), 6.88(\mathrm{dd}, J=8.08,1.16$, $1 \mathrm{H}), 6.81(\mathrm{~d}, J=8.08,1 \mathrm{H}), 6.36(\mathrm{~d}, J=2.08,1 \mathrm{H}), 6.32(\mathrm{~d}$, $J=2.44,1 \mathrm{H}), 5.96(\mathrm{~d}, J=6.40,1 \mathrm{H}), 5.23(\mathrm{~d}, J=11.60$, $1 \mathrm{H}), 4.98(\mathrm{t}, J=5.40,1 \mathrm{H}), 4.92(\mathrm{~d}, J=7.92,1 \mathrm{H})$, 4.79-4.73 (m, 1H), 4.19-4.16 (m, 1H), $3.78(\mathrm{~s}, 3 \mathrm{H})$, 3.56-3.51 (m, 1H), 3.37-3.31 (m, 1H), $3.00(\mathrm{~s}, 3 \mathrm{H}), 2.90(\mathrm{~s}$, $3 \mathrm{H}$ ). ${ }^{13} \mathrm{C}$ NMR (DMSO- $\left.d_{6}, 126 \mathrm{MHz}\right) \delta 199.8,162.3$, $162.1,159.4,153.0,148.0,147.4,144.2,143.7,130.2$, $127.8,121.9,120.9,117.1,116.8,115.7,112.0,104.6$, 103.0, 101.8, 83.1, 78.5, 76.3, 72.1, 60.6, 56.0, 36.8, 36.6. $\mathrm{MS}\left(\mathrm{ESI}^{-}\right) \mathrm{m} / z$ calcd for $\mathrm{C}_{28} \mathrm{H}_{26} \mathrm{NO}_{11} 552.15$, found 552.30 $(\mathrm{M}-\mathrm{H})^{-}$. 
(2R,3R)-3,5-dihydroxy-2-(3-(4-hydroxy-3-methoxyphenyl)-2(hydroxymethyl)-2,3-dihydrobenzo[b][1,4]dioxin-6-yl)-4oxochroman-7-yl 4-methylpiperazine-1-carboxylate (2c)

White solid (53.0 mg, purity $95.03 \%$, yield $17.4 \%$ ); mp $137-140{ }^{\circ} \mathrm{C}$; ${ }^{1} \mathrm{H}$ NMR (DMSO- $\left.d_{6}, 400 \mathrm{MHz}\right) \delta 11.68(\mathrm{~s}$, $1 \mathrm{H}), 9.16(\mathrm{~s}, 1 \mathrm{H}), 7.11(\mathrm{~d}, J=2.16,1 \mathrm{H}), 7.04(\mathrm{~d}, 1 \mathrm{H}), 7.02$ $(\mathrm{s}, 1 \mathrm{H}), 6.99(\mathrm{~d}, J=8.72,1 \mathrm{H}), 6.88(\mathrm{dd}, J=8.23,1.52$, $1 \mathrm{H}), 6.81(\mathrm{~d}, J=8.08,1 \mathrm{H}), 6.37(\mathrm{~d}, J=2.08,1 \mathrm{H}), 6.33(\mathrm{~d}$, $J=2.44,1 \mathrm{H}), 5.96(\mathrm{~d}, J=6.40,1 \mathrm{H}), 5.23(\mathrm{~d}, J=11.52$, $1 \mathrm{H}), 4.98(\mathrm{t}, J=5.56,1 \mathrm{H}), 4.92(\mathrm{~d}, J=7.96,1 \mathrm{H})$, 4.79-4.73 (m, 1H), 4.20-4.16 (m, 1H), 3.78 (s, 3H), 3.53 (br, 3H), 3.41 (br, 2H), $3.35(\mathrm{~m}, 1 \mathrm{H}), 2.35$ (br, 4H), 2.21 (s, $3 \mathrm{H}) .{ }^{13} \mathrm{C}$ NMR (DMSO- $\left.d_{6}, 126 \mathrm{MHz}\right) \delta 199.8,162.3$, $162.1,159.3,151.9,148.0,147.4,144.2,143.7,130.2$, $127.9,121.7,120.9,117.1,116.8,115.7,112.0,104.7$, 102.9, 101.7, 83.1, 78.5, 76.3, 72.5, 60.6, 56.1, 54.5, 54.3, 46.0, 44.6, 44.0. $\mathrm{MS}\left(\mathrm{ESI}^{+}\right) \mathrm{m} / \mathrm{z}$ calcd for $\mathrm{C}_{31} \mathrm{H}_{33} \mathrm{~N}_{2} \mathrm{O}_{11}$ 609.21 , found $609.40(\mathrm{M}+\mathrm{H})^{+}$.

(2R,3R)-3,5-dihydroxy-2-(3-(4-hydroxy-3-methoxyphenyl)-2(hydroxymethyl)-2,3-dihydrobenzo[b][1,4]dioxin-6-yl)-4oxochroman-7-yl diethylcarbamate (2d)

White solid (140.6 mg, purity $97.90 \%$, yield $48.4 \%$ ); $\mathrm{mp}$ 129-132 ${ }^{\circ} \mathrm{C}$; ${ }^{1} \mathrm{H}$ NMR (DMSO- $\left.d_{6}, 400 \mathrm{MHz}\right) \delta 11.68$ (s, $1 \mathrm{H}), 9.16(\mathrm{~s}, 1 \mathrm{H}), 7.11(\mathrm{~d}, J=2.24,1 \mathrm{H}), 7.07(\mathrm{~d}, 1 \mathrm{H}), 7.02$ $(\mathrm{d}, J=1.36,1 \mathrm{H}), 7.00(\mathrm{~d}, J=8.88,1 \mathrm{H}), 6.88(\mathrm{dd}, J=8.12$, $1.64,1 \mathrm{H}), 6.81(\mathrm{~d}, J=8.08,1 \mathrm{H}), 6.35(\mathrm{~d}, J=2.08,1 \mathrm{H})$, $6.31(\mathrm{~d}, J=2.32,1 \mathrm{H}), 5.96(\mathrm{~d}, J=6.32,1 \mathrm{H}), 5.23(\mathrm{~d}, J=$ $11.60,1 \mathrm{H}), 4.97(\mathrm{t}, J=1.00,1 \mathrm{H}), 4.92(\mathrm{~d}, J=7.92,1 \mathrm{H})$, 4.79-4.73 (m, 1H), 4.20-4.16 (m, 1H), $3.78(\mathrm{~s}, 3 \mathrm{H})$, $3.52-3.55$ (m, 1H), 3.35-3.33 (m, 1H), 3.31-3.26 (q, $J=$ $6.76,4 \mathrm{H}), 1.17(\mathrm{t}, J=6.92,3 \mathrm{H}), 1.12(\mathrm{t}, J=6.92,3 \mathrm{H}) .{ }^{13} \mathrm{C}$ NMR (DMSO- $\left.d_{6}, 126 \mathrm{MHz}\right) \delta 199.8,162.3,162.1,159.5$, $152.3,148.0,147.4,144.2,143.7,130.2,127.8,121.9$, $120.9,117.1,116.8,115.7,112.0,104.6,102.8,101.6$, 83.1, 78.5, 76.3, 72.2, 60.6, 56.0, 42.3, 42.1, 14.6, 13.6. MS $\left(\mathrm{ESI}^{+}\right) \mathrm{m} / z$ calcd for $\mathrm{C}_{30} \mathrm{H}_{31} \mathrm{NO}_{11} \mathrm{Na} 604.18$, found 604.40 $(\mathrm{M}+\mathrm{Na})^{+}$.

(2R,3R)-3,5-dihydroxy-2-(3-(4-hydroxy-3-methoxyphenyl)-2(hydroxymethyl)-2,3-dihydrobenzo[b][1,4]dioxin-6-yl)-4oxochroman-7-yl pyrrolidine-1-carboxylate (2e)

White solid (75.6 mg, purity $95.75 \%$, yield $26.1 \%$ ); mp $142-145^{\circ} \mathrm{C} ;{ }^{1} \mathrm{H}$ NMR (DMSO- $\left.d_{6}, 400 \mathrm{MHz}\right) \delta 11.68(\mathrm{~s}$, $1 \mathrm{H}), 9.16(\mathrm{~s}, 1 \mathrm{H}), 7.11(\mathrm{~d}, J=2.161 \mathrm{H}), 7.06(\mathrm{~d}, 1 \mathrm{H}), 7.02$ $(\mathrm{d}, J=1.52,1 \mathrm{H}), 6.99(\mathrm{~d}, J=8.92,1 \mathrm{H}), 6.88(\mathrm{dd}, J=8.16$, $1.24,1 \mathrm{H}), 6.81(\mathrm{~d}, J=8.08,1 \mathrm{H}), 6.37(\mathrm{~d}, J=2.08,1 \mathrm{H})$, $6.33(\mathrm{~d}, J=2.52,1 \mathrm{H}), 5.96(\mathrm{~d}, J=6.36,1 \mathrm{H}), 5.23(\mathrm{~d}, J=$
$11.56,1 \mathrm{H}), 4.98(\mathrm{t}, J=5.44,1 \mathrm{H}), 4.92(\mathrm{~d}, J=7.96,1 \mathrm{H})$, 4.79-4.73 (m, 1H), 4.19-4.17 (m, 1H), $3.78(\mathrm{~s}, 3 \mathrm{H})$, $3.56-3.51(\mathrm{~m}, 1 \mathrm{H}), 3.46-3.43(\mathrm{t}, J=6.32,2 \mathrm{H}), 3.37-3.33$ $(\mathrm{m}, 1 \mathrm{H}), 3.32(\mathrm{t}, J=6.12,2 \mathrm{H}), 1.89-1.82(\mathrm{~m}, 4 \mathrm{H}) .{ }^{13} \mathrm{C}$ NMR (DMSO- $\left.d_{6}, 126 \mathrm{MHz}\right) \delta 199.9,162.3,162.1,159.3$, $151.2,148.0,147.4,144.2,143.7,130.2,127.8,121.9$, $120.9,117.1,116.8,115.7,112.0,104.6,102.9,101.7$, 83.1, 78.5, 76.3, 72.1, 60.6, 56.0, 46.7, 46.6, 25.6, 24.8. MS $\left(\mathrm{ESI}^{+}\right) \mathrm{m} / z$ calcd for $\mathrm{C}_{30} \mathrm{H}_{29} \mathrm{NO}_{11} \mathrm{Na} 602.16$, found 602.30 $(\mathrm{M}+\mathrm{Na})^{+}$.

(2R,3R)-3,5-dihydroxy-2-(3-(4-hydroxy-3-methoxyphenyl)-2(hydroxymethyl)-2,3-dihydrobenzo[b][1,4]dioxin-6-yl)-4oxochroman-7-yl piperidine-1-carboxylate (2f)

White solid (138.0 mg, purity $97.99 \%$, yield $46.5 \%$ ); $\mathrm{mp}$ $146-147{ }^{\circ} \mathrm{C} ;{ }^{1} \mathrm{H}$ NMR (DMSO- $\left.d_{6}, 400 \mathrm{MHz}\right) \delta 11.68(\mathrm{~s}$, $1 \mathrm{H}), 9.16(\mathrm{~s}, 1 \mathrm{H}), 7.11(\mathrm{~d}, J=2.00,1 \mathrm{H}), 7.04(\mathrm{~d}, 1 \mathrm{H})$, $7.02(\mathrm{~s}, 1 \mathrm{H}), 6.99(\mathrm{~d}, J=8.72,1 \mathrm{H}), 6.88(\mathrm{dd}, J=8.12$, $1.44,1 \mathrm{H}), 6.81(\mathrm{~d}, J=8.04,1 \mathrm{H}), 6.35(\mathrm{~d}, J=2.08,1 \mathrm{H})$, $6.32(\mathrm{~d}, J=2.44,1 \mathrm{H}), 5.96(\mathrm{~d}, J=6.36,1 \mathrm{H}), 5.23(\mathrm{~d}, J=$ $11.56,1 \mathrm{H}), 4.98(\mathrm{t}, J=5.44,1 \mathrm{H}), 4.92(\mathrm{~d}, J=7.96,1 \mathrm{H})$, 4.79-4.73 (m, 1H), 4.19-4.16 (m, 1H), $3.78(\mathrm{~s}, 3 \mathrm{H})$, 3.56-3.51 (m, 1H), 3.49 (br, 2H), 3.39 (br, 2H), 3.37-3.31 $(\mathrm{m}, 1 \mathrm{H}), 1.58-1.53(\mathrm{~m}, 6 \mathrm{H}) .{ }^{13} \mathrm{C}$ NMR (DMSO- $d_{6}$, $126 \mathrm{MHz}) \delta 199.9,162.3,162.1,159.5,151.8,148.0$, $147.4,144.2,143.7,130.2,127.8,121.7,120.9,117.0$, $116.8,115.7,111.9,104.6,102.9,101.7,83.1,78.5,76.3$, 72.1, 60.6, 56.0, 45.7, 45.1, 25.8, 25.4, 24.0. MS(ESI $\left.{ }^{+}\right)$ $m / z$ calcd for $\mathrm{C}_{31} \mathrm{H}_{31} \mathrm{NO}_{11} \mathrm{Na} 616.18$, found 616.30 $(\mathrm{M}+\mathrm{Na})^{+}$.

(2R,3R)-3,5-dihydroxy-2-(3-(4-hydroxy-3-methoxyphenyl)-2(hydroxymethyl)-2,3-dihydrobenzo[b][1,4]dioxin-6-yl)-4oxochroman-7-yl piperidine-1-carboxylate (2g)

White solid (121.0 mg, purity $98.32 \%$, yield $39.7 \%$ ); mp 138-141 ${ }^{\circ} \mathrm{C}$; ${ }^{1} \mathrm{H}$ NMR (DMSO-d $\left.6,400 \mathrm{MHz}\right) \delta 11.69(\mathrm{~s}$, 1H), 9.16 (s, 1H), 7.12 (d, $J=1.00,1 \mathrm{H}), 7.05(\mathrm{~d}, 1 \mathrm{H}), 7.02$ (s, 1H), $7.00(\mathrm{dd}, J=8.36,0.68,1 \mathrm{H}), 6.88(\mathrm{dd}, J=8.16$, $1.68,1 \mathrm{H}), 6.81(\mathrm{~d}, J=8.08,1 \mathrm{H}), 6.32(\mathrm{~d}, J=2.08,1 \mathrm{H})$, $6.28(\mathrm{~d}, J=2.32,1 \mathrm{H}), 5.96(\mathrm{~d}, J=6.36,1 \mathrm{H}), 5.23(\mathrm{~d}, J=$ $11.60,1 \mathrm{H}), 4.98(\mathrm{t}, J=4.92,1 \mathrm{H}), 4.92(\mathrm{~d}, J=7.92,1 \mathrm{H})$, 4.78-4.73 (m, 1H), 4.20-4.16 (m, 1H), 3.91 (br, 2H), 3.78 (s, 3H), 3.55-3.52 (m, 1H), 3.37-3.31 (m, 1H), 1.22 (br, 12H). ${ }^{13} \mathrm{C}$ NMR (DMSO- $\left.d_{6}, 126 \mathrm{MHz}\right) \delta 199.8,162.4$, $162.2,159.5,151.8,148.0,147.4,144.2,143.7,130.2$, $127.8,121.8,120.9,117.1,116.8,115.7,112.0,104.4$, $102.4,101.3,83.1,78.5,76.3,72.2,60.6,56.0,47.0,46.4$, 21.7, 20.4. $\mathrm{MS}\left(\mathrm{ESI}^{+}\right) \mathrm{m} / z$ calcd for $\mathrm{C}_{32} \mathrm{H}_{35} \mathrm{NO}_{11} \mathrm{Na} 632.21$, found $632.40(\mathrm{M}+\mathrm{Na})^{+}$. 
(2R,3R)-3,5-dihydroxy-2-(3-(4-hydroxy-3-methoxyphenyl)-2(hydroxymethyl)-2,3-dihydrobenzo[b][1,4]dioxin-6-yl)-4oxochroman-7-yl [1,4'-bipiperidine]-1'-carboxylate (2h)

White solid (37.7 mg, purity $98.22 \%$, yield $10.8 \%$ ); mp 146-149 ${ }^{\circ} \mathrm{C}$; ${ }^{1} \mathrm{H}$ NMR (DMSO- $\left.d_{6}, 400 \mathrm{MHz}\right) \delta 11.69$ (s, $1 \mathrm{H}), 9.21(\mathrm{~s}, 1 \mathrm{H}), 7.11(\mathrm{~s}, 1 \mathrm{H}), 7.06-702(\mathrm{~m}, 2 \mathrm{H}), 7.00(\mathrm{~d}$, $J=6.96,1 \mathrm{H}), 6.87(\mathrm{dd}, J=6.32,1 \mathrm{H}), 6.81(\mathrm{~d}, J=6.32$, $1 \mathrm{H}), 6.37(\mathrm{~s}, 1 \mathrm{H}), 6.33(\mathrm{~s}, 1 \mathrm{H}), 5.99$ (d, $J=4.60,1 \mathrm{H}), 5.23$ $(\mathrm{d}, J=9.24,1 \mathrm{H}), 5.00(\mathrm{br}, 1 \mathrm{H}), 4.92(\mathrm{~d}, J=6.44,1 \mathrm{H})$, 4.79-4.75 (m, 1H), 4.18 (br, 1H), 4.10 (br, 1H), 4.03 (br, $1 \mathrm{H}), 3.78(\mathrm{~s}, 3 \mathrm{H}), 3.55-3.53(\mathrm{~m}, 1 \mathrm{H}), 3.53-3.35(\mathrm{~m}, 3 \mathrm{H})$, $3.00(\mathrm{t}, J=10.04,1 \mathrm{H}), 2.87(\mathrm{t}, J=9.92,1 \mathrm{H}), 2.50(\mathrm{~m}, 3 \mathrm{H})$, 1.79-1.76 (m, 2H), 1.50-1.39 (m, 8H). ${ }^{13} \mathrm{C}$ NMR (DMSO$\left.d_{6}, 126 \mathrm{MHz}\right) \delta 199.8,162.3,162.1,159.4,151.8,148.0$, $147.4,144.2,143.7,130.2,127.8,121.9,120.9,117.1$, $116.8,115.7,111.9,104.6,103.0,101.8,83.1,78.5,76.3$, 72.1, 61.7, 60.5, 56.0, 50.0, 44.3, 43.8, 27.8, 27.3, 26.1, 24.6. $\mathrm{MS}\left(\mathrm{ESI}^{+}\right) \mathrm{m} / \mathrm{z}$ calcd for $\mathrm{C}_{36} \mathrm{H}_{41} \mathrm{~N}_{2} \mathrm{O}_{11} 677.27$, found $677.60(\mathrm{M}+\mathrm{H})^{+}$.

(2R,3R)-3,5-dihydroxy-2-(3-(4-hydroxy-3-methoxyphenyl)-2(hydroxymethyl)-2,3-dihydrobenzo[b][1,4]dioxin-6-yl)-4oxochroman-7-yl methoxy(methyl)carbamate (2i)

White solid (86.0 mg, purity $97.34 \%$, yield $30.2 \%$ ); $\mathrm{mp}$ $178-181{ }^{\circ} \mathrm{C} ;{ }^{1} \mathrm{H}$ NMR (DMSO- $\left.d_{6}, 400 \mathrm{MHz}\right) \delta 11.69(\mathrm{~s}$, $1 \mathrm{H}), 9.16(\mathrm{~s}, 1 \mathrm{H}), 7.12(\mathrm{~d}, J=1.92,1 \mathrm{H}), 7.07-7.02(\mathrm{~m}$, $2 \mathrm{H}), 7.00(\mathrm{~d}, J=8.64,1 \mathrm{H}), 6.88(\mathrm{dd}, J=8.32,1.16,1 \mathrm{H})$, $6.81(\mathrm{~d}, J=8.08,1 \mathrm{H}), 6.43(\mathrm{~d}, J=2.08,1 \mathrm{H}), 6.39(\mathrm{~d}, J=$ $2.32,1 \mathrm{H}), 5.98(\mathrm{~d}, J=6.36,1 \mathrm{H}), 5.25(\mathrm{~d}, J=11.64,1 \mathrm{H})$, $4.98(\mathrm{t}, J=5.44,1 \mathrm{H}), 4.92(\mathrm{~d}, J=7.96,1 \mathrm{H}), 4.81-4.76(\mathrm{~m}$, $1 \mathrm{H}), 4.19-4.17(\mathrm{~m}, 1 \mathrm{H}), 3.78(\mathrm{~s}, 3 \mathrm{H}), 3.71(\mathrm{~s}, 3 \mathrm{H})$, 3.56-3.52 (m, 1H), 3.37-3.31 (m, 1H), $3.21(\mathrm{~s}, 3 \mathrm{H}) .{ }^{13} \mathrm{C}$ NMR (DMSO- $\left.d_{6}, 126 \mathrm{MHz}\right) \delta 199.9,162.3,162.2,158.6$, $153.0,148.0,147.4,144.2,143.7,130.1,127.9,121.7$, $120.9,117.1,116.8,115.7,112.0,105.0,103.0,101.9$,

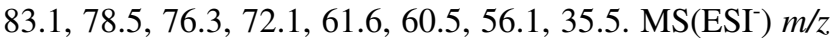
calcd for $\mathrm{C}_{28} \mathrm{H}_{26} \mathrm{NO}_{12} 568.15$, found $568.30(\mathrm{M}-\mathrm{H})^{-}$.

\section{General procedure for the synthesis of compounds 3a-i}

To a solution of silibinin $(241.2 \mathrm{mg}, 0.50 \mathrm{mmol}, 1.0 \mathrm{eq})$ in DMF (3.6 mL, 15.0 vol) was added $\mathrm{K}_{2} \mathrm{CO}_{3}$ (69.2 mg, $0.50 \mathrm{mmol}, 1.0 \mathrm{eq})$ and carbamyl chloride $\mathbf{1 a}-\mathbf{i}$ $(0.53 \mathrm{mmol}, 1.05 \mathrm{eq})$. The mixture was stirred at $25^{\circ} \mathrm{C}$ under $\mathrm{N}_{2}$ atmosphere for $12 \mathrm{~h}$. After completion of the reaction (monitored by TLC), the reaction mixture diluted with water $(4.0 \mathrm{~mL})$ and the resulting precipitate was collected, washed with water. The solid was purified by column chromatography $\left(\mathrm{CH}_{2} \mathrm{Cl}_{2} / \mathrm{MeOH}=15: 1\right)$ to give the title compound $\mathbf{3 a - i}$. 3,5-dihydroxy-2-(3-(4-hydroxy-3-methoxyphenyl)-2(hydroxymethyl)-2,3-dihydrobenzo [b][1,4]dioxin-6-yl)-4oxo-4H-chromen-7-yl morpholine-4-carboxylate (3a)

Yellow solid (81.8 mg, purity $98.19 \%$, yield $27.3 \%$ ); $\mathrm{mp}$ 284-286 ${ }^{\circ} \mathrm{C}$; ${ }^{1} \mathrm{H}$ NMR (DMSO-d $\left.6,400 \mathrm{MHz}\right) \delta 12.46(\mathrm{~s}$, $1 \mathrm{H}), 9.90(\mathrm{~s}, 1 \mathrm{H}), 9.18(\mathrm{~s}, 1 \mathrm{H}), 7.85(\mathrm{dd}, J=10.68,2.16$, $1 \mathrm{H}), 7.84(\mathrm{~s}, 1 \mathrm{H}), 7.16(\mathrm{~d}, J=8.40,1 \mathrm{H}), 7.11(\mathrm{~d}, J=2.00$, $1 \mathrm{H}), 7.06(\mathrm{~d}, J=1.76,1 \mathrm{H}), 6.91(\mathrm{dd}, J=8.20,1.76,1 \mathrm{H})$, $6.83(\mathrm{~d}, J=8.04,1 \mathrm{H}), 6.64(\mathrm{~d}, J=2.04,1 \mathrm{H}), 5.03(\mathrm{t}, J=$ $5.44,1 \mathrm{H}), 4.99(\mathrm{~d}, J=7.92,1 \mathrm{H}), 4.31-4.28(\mathrm{~m}, 1 \mathrm{H}), 3.79$ (s, 3H), $3.67(\mathrm{t}, J=5.04,4 \mathrm{H}), 3.59-3.56(\mathrm{~m}, 3 \mathrm{H}), 3.44(\mathrm{br}$, 2H), 3.40-3.36 (m, 1H). ${ }^{13} \mathrm{C}$ NMR (DMSO- $\left.d_{6}, 126 \mathrm{MHz}\right) \delta$ $176.9,160.3,156.6,155.2,152.3,148.0,147.5,147.4$, $145.8,143.9,137.5,127.6,123.9,122.0,121.0,117.3$, 116.9, 115.7, 112.0, 107.3, 104.3, 101.5, 79.0, 76.3, 66.1, 60.5, 56.1, 45.1, 44.3. MS(ESI $\left.{ }^{+}\right) \mathrm{m} / z$ calcd for $\mathrm{C}_{30} \mathrm{H}_{28} \mathrm{NO}_{12}$ 594.16, found $594.4(\mathrm{M}+\mathrm{H})^{+}$.

\section{3,5-dihydroxy-2-(3-(4-hydroxy-3-methoxyphenyl)-2-} (hydroxymethyl)-2,3-dihydrobenzo[b][1,4]dioxin-6-yl)-4oxo-4H-chromen-7-yl dimethylcarbamate (3b)

Yellow solid (109.9 mg, purity $95.75 \%$, yield $39.9 \%$ ); mp $134-137{ }^{\circ} \mathrm{C}$; ${ }^{1} \mathrm{H}$ NMR (DMSO- $\left.d_{6}, 400 \mathrm{MHz}\right) \delta 12.45(\mathrm{~s}$, $1 \mathrm{H}), 9.88$ (s, 1H), $9.18(\mathrm{~s}, 1 \mathrm{H}), 7.84-7.81(\mathrm{~m}, 2 \mathrm{H}), 7.16(\mathrm{~d}$, $J=8.44,1 \mathrm{H}), 7.07(\mathrm{~d}, J=2.04,1 \mathrm{H}), 7.05(\mathrm{~d}, J=1.72$, $1 \mathrm{H}), 6.90(\mathrm{dd}, J=8.16,1.80,1 \mathrm{H}), 6.82(\mathrm{~d}, J=8.08,1 \mathrm{H})$, $6.60(\mathrm{~d}, J=2.04,1 \mathrm{H}), 5.03(\mathrm{t}, J=5.48,1 \mathrm{H}), 4.99(\mathrm{~d}, J=$ 7.92, 1H), 4.31-4.27 (m, 1H), $3.79(\mathrm{~s}, 3 \mathrm{H}), 3.60-3.55(\mathrm{~m}$, 1H), 3.40-3.33 (m, 1H), 3.05 (s, 3H), 2.93 (s, 3H). ${ }^{13} \mathrm{C}$ NMR (DMSO- $\left.d_{6}, 126 \mathrm{MHz}\right) \delta 176.9,160.3,156.8,155.2$, $153.3,148.0,147.4,147.4,145.8,143.9,137.5,127.6$, $123.9,122.0,121.0,117.3,116.9,115.7,112.0,107.2$, 104.3, 101.4, 79.0, 76.3, 60.5, 56.0, 36.8, 36.6. MS(ESI $\left.{ }^{+}\right)$ $m / z$ calcd for $\mathrm{C}_{28} \mathrm{H}_{26} \mathrm{NO}_{11} 552.15$, found $552.40(\mathrm{M}+\mathrm{H})^{+}$.

\section{3,5-dihydroxy-2-(3-(4-hydroxy-3-methoxyphenyl)-2-} (hydroxymethyl)-2,3-dihydrobenzo[b][1,4]dioxin-6-yl)-4oxo-4H-chromen-7-yl 4-methylpiperazine-1-carboxylate (3c)

Yellow solid (63.0 mg, purity $97.83 \%$, yield $20.8 \%$ ); mp $198-201{ }^{\circ} \mathrm{C}$; ${ }^{1} \mathrm{H}$ NMR (DMSO- $\left.d_{6}, 400 \mathrm{MHz}\right) \delta 12.45$ (s, $1 \mathrm{H}), 9.90(\mathrm{~s}, 1 \mathrm{H}), 9.18(\mathrm{~s}, 1 \mathrm{H}), 7.84-7.82(\mathrm{~m}, 2 \mathrm{H}), 7.15(\mathrm{~d}$, $J=8.48,1 \mathrm{H}), 7.09(\mathrm{~d}, J=2.04,1 \mathrm{H}), 7.05(\mathrm{~d}, J=1.68$, $1 \mathrm{H}), 6.90(\mathrm{dd}, J=8.12,1.72,1 \mathrm{H}), 6.82(\mathrm{~d}, J=8.08,1 \mathrm{H})$, $6.61(\mathrm{~d}, J=2.04,1 \mathrm{H}), 5.03(\mathrm{t}, J=5.36,1 \mathrm{H}), 4.99(\mathrm{~d}, J=$ 7.88, 1H), 4.31-4.27 (m, 1H), $3.79(\mathrm{~s}, 3 \mathrm{H}), 3.58(\mathrm{br}, 3 \mathrm{H})$, 3.44 (br, 2H), 3.40-3.35 (m, 1H), 2.38 (br, 4H), 2.23 (s, $3 \mathrm{H}) .{ }^{13} \mathrm{C}$ NMR (DMSO- $\left.d_{6}, 126 \mathrm{MHz}\right) \delta 176.9,160.3$, $156.7,155.2,152.2,148.1,147.5,147.4,145.8,143.9$, $137.5,127.6,123.9,122.0,121.0,117.3,116.9,115.7$, 
$112.0,107.3,104.2,101.5,79.0,76.3,60.5,56.1,54.6$, 54.4, 46.1, 44.7, 44.0. MS(ESI $\left.{ }^{+}\right) \mathrm{m} / z$ calcd for $\mathrm{C}_{31} \mathrm{H}_{31} \mathrm{~N}_{2} \mathrm{O}_{11}$ 607.19, found $607.30(\mathrm{M}+\mathrm{H})^{+}$.

\section{3,5-dihydroxy-2-(3-(4-hydroxy-3-methoxyphenyl)-2- (hydroxymethyl)-2,3-dihydrobenzo[b][1,4]dioxin-6-yl)-4- oxo-4H-chromen-7-yl diethylcarbamate (3d)}

Yellow solid (53.2 mg, purity $97.62 \%$, yield $18.4 \%$ ); mp $224-227{ }^{\circ} \mathrm{C}$; ${ }^{1} \mathrm{H}$ NMR (DMSO- $\left.d_{6}, 400 \mathrm{MHz}\right) \delta 12.44(\mathrm{~s}$, $1 \mathrm{H}), 9.89(\mathrm{~s}, 1 \mathrm{H}), 9.18(\mathrm{~s}, 1 \mathrm{H}), 7.86-7.83(\mathrm{~m}, 2 \mathrm{H}), 7.15(\mathrm{~d}$, $J=9.16,1 \mathrm{H}), 7.09(\mathrm{~d}, J=2.04,1 \mathrm{H}), 7.05(\mathrm{~d}, J=1.76$, $1 \mathrm{H}), 6.90(\mathrm{dd}, J=8.16,1.76,1 \mathrm{H}), 6.82(\mathrm{~d}, J=8.08,1 \mathrm{H})$, $6.60(\mathrm{~d}, J=2.04,1 \mathrm{H}), 5.03(\mathrm{t}, J=5.36,1 \mathrm{H}), 4.99(\mathrm{~d}, J=$ 7.92, 1H), 4.31-4.27 (m, 1H), $3.79(\mathrm{~s}, 3 \mathrm{H}), 3.60-3.55(\mathrm{~m}$, $1 \mathrm{H}), 3.42-3.35(\mathrm{~m}, 3 \mathrm{H}), 3.33-3.28(\mathrm{~m}, 2 \mathrm{H}), 1.21(\mathrm{t}, J=$ $7.04,3 \mathrm{H}$ ), $1.15(\mathrm{t}, J=7.00,3 \mathrm{H}) .{ }^{13} \mathrm{C}$ NMR (DMSO- $d_{6}$, $126 \mathrm{MHz}) \delta 176.9,160.3,156.8,155.3,152.6,148.0$, $147.4,147.4,145.8,143.9,137.5,127.6,123.9,122.0$, $121.0,117.3,116.9,115.7,112.0,107.2,104.2,101.5$, 79.0, 76.3, 60.5, 56.0, 42.3, 42.1, 14.6, 13.6. $\mathrm{MS}\left(\mathrm{ESI}^{+}\right) \mathrm{m} / \mathrm{z}$ calcd for $\mathrm{C}_{30} \mathrm{H}_{30} \mathrm{NO}_{11} 580.18$, found $580.30(\mathrm{M}+\mathrm{H})^{+}$.

\section{3,5-dihydroxy-2-(3-(4-hydroxy-3-methoxyphenyl)-2- (hydroxymethyl)-2,3-dihydrobenzo[b][1,4]dioxin-6-yl)-4- oxo-4H-chromen-7-yl pyrrolidine-1-carboxylate (3e)}

Yellow solid (52.6 mg, purity $98.66 \%$, yield $18.2 \%$ ); mp $133-136{ }^{\circ} \mathrm{C}$; ${ }^{1} \mathrm{H}$ NMR (DMSO- $\left.d_{6}, 400 \mathrm{MHz}\right) \delta 12.44(\mathrm{~s}$, $1 \mathrm{H}), 9.88(\mathrm{~s}, 1 \mathrm{H}), 9.18(\mathrm{~s}, 1 \mathrm{H}), 7.84-7.81(\mathrm{~m}, 2 \mathrm{H}), 7.15(\mathrm{~d}$, $J=9.16,1 \mathrm{H}), 7.08(\mathrm{~d}, J=2.00,1 \mathrm{H}), 7.05(\mathrm{~d}, J=1.72$, $1 \mathrm{H}), 6.90(\mathrm{dd}, J=8.16,1.76,1 \mathrm{H}), 6.82(\mathrm{~d}, J=8.04,1 \mathrm{H})$, $6.61(\mathrm{~d}, J=2.04,1 \mathrm{H}), 5.03(\mathrm{t}, J=5.44,1 \mathrm{H}), 4.99(\mathrm{~d}, J=$ 7.92, 1H), 4.31-4.27 (m, 1H), $3.79(\mathrm{~s}, 3 \mathrm{H}), 3.60-3.55(\mathrm{~m}$, $1 \mathrm{H}), 3.52(\mathrm{t}, J=6.52,2 \mathrm{H}), 3.39-3.36(\mathrm{~m}, 3 \mathrm{H}), 1.92-1.85$ $(\mathrm{m}, 4 \mathrm{H}) .{ }^{13} \mathrm{C}$ NMR (DMSO- $\left.d_{6}, 126 \mathrm{MHz}\right) \delta 176.9,160.3$, $156.7,155.3,151.4,148.0,147.5,147.4,145.8,143.9$, $137.5,127.6,123.9,122.0,121.0,117.3,116.9,115.7$, 112.0, 107.2, 104.2, 101.4, 79.0, 76.3, 60.5, 56.1, 46.8, 46.6, 25.7, 24.9. $\mathrm{MS}\left(\mathrm{ESI}^{+}\right) \mathrm{m} / \mathrm{z}$ calcd for $\mathrm{C}_{30} \mathrm{H}_{28} \mathrm{NO}_{11}$ 578.17, found $578.40(\mathrm{M}+\mathrm{H})^{+}$.

(2R,3R)-3,5-dihydroxy-2-(3-(4-hydroxy-3-methoxyphenyl)-2(hydroxymethyl)-2,3-dihydrobenzo[b][1,4]dioxin-6-yl)-4oxochroman-7-yl piperidine-1-carboxylate (3f)

Yellow solid (67.8 mg, purity $97.07 \%$, yield $22.9 \%$ ); mp $132-135{ }^{\circ} \mathrm{C}$; ${ }^{1} \mathrm{H}$ NMR (DMSO- $\left.d_{6}, 400 \mathrm{MHz}\right) \delta 12.44(\mathrm{~s}$, $1 \mathrm{H}), 9.89(\mathrm{~s}, 1 \mathrm{H}), 9.18(\mathrm{~s}, 1 \mathrm{H}), 7.85-7.82(\mathrm{~m}, 2 \mathrm{H}), 7.15(\mathrm{~d}$, $J=8.28,1 \mathrm{H}), 7.08(\mathrm{~d}, J=1.16,1 \mathrm{H}), 7.05(\mathrm{~d}, J=1.64,1 \mathrm{H})$, $6.90(\mathrm{dd}, J=8.16,1.72,1 \mathrm{H}), 6.82(\mathrm{~d}, J=8.04,1 \mathrm{H}), 6.60(\mathrm{~d}$, $J=1.72,1 \mathrm{H}), 5.03(\mathrm{t}, J=5.40,1 \mathrm{H}), 4.99(\mathrm{~d}, J=7.92,1 \mathrm{H})$, $4.31-4.27(\mathrm{~m}, 1 \mathrm{H}), 3.79(\mathrm{~s}, 3 \mathrm{H}), 3.60-3.55(\mathrm{~m}, 3 \mathrm{H})$,
3.42-3.35 (m, 3H), $1.56(\mathrm{br}, 6 \mathrm{H}) .{ }^{13} \mathrm{C}$ NMR (DMSO- $d_{6}$, $126 \mathrm{MHz}) \delta 176.9,160.3,156.9,155.3,152.1,148.0,147.4$, $147.4,145.8,143.9,137.5,127.6,123.9,122.0,121.0$, $117.3,116.9,115.7,112.0,107.2,104.3,101.5,79.0,76.3$, 60.5, 56.0, 45.7, 45.1, 25.9, 25.5, 24.1. MS(ESI $\left.{ }^{+}\right) \mathrm{m} / z$ calcd for $\mathrm{C}_{31} \mathrm{H}_{30} \mathrm{NO}_{11} 592.18$, found $592.40(\mathrm{M}+\mathrm{H})^{+}$.

\section{3,5-dihydroxy-2-(3-(4-hydroxy-3-methoxyphenyl)-2-} (hydroxymethyl)-2,3-dihydrobenzo[b][1,4]dioxin-6-yl)-4oxo-4H-chromen-7-yl diisopropylcarbamate (3g)

Yellow solid (34.1 mg, purity $97.55 \%$, yield $11.2 \%$ ); mp $125-128{ }^{\circ} \mathrm{C}$; ${ }^{1} \mathrm{H}$ NMR (DMSO-d $\left.d_{6}, 400 \mathrm{MHz}\right) \delta 12.44$ (s, $1 \mathrm{H}), 9.89(\mathrm{~s}, 1 \mathrm{H}), 9.18(\mathrm{~s}, 1 \mathrm{H}), 7.88-7.85(\mathrm{~m}, 2 \mathrm{H}), 7.15(\mathrm{dd}$, $J=6.32,2.84,1 \mathrm{H}), 7.07(\mathrm{~d}, J=2.04,1 \mathrm{H}), 7.05(\mathrm{~d}, J=$ $1.76,1 \mathrm{H}), 6.90(\mathrm{dd}, J=8.12,1.72,1 \mathrm{H}), 6.82(\mathrm{~d}, J=8.08$, $1 \mathrm{H}), 6.57(\mathrm{~d}, J=2.04,1 \mathrm{H}), 5.02(\mathrm{t}, J=5.48,1 \mathrm{H}), 4.98(\mathrm{~d}$, $J=7.92,1 \mathrm{H}), 4.31-4.28(\mathrm{~m}, 1 \mathrm{H}), 3.99(\mathrm{br}, 2 \mathrm{H}), 3.79(\mathrm{~s}$, $3 \mathrm{H}), 3.60-3.55(\mathrm{~m}, 1 \mathrm{H}), 3.40-3.33(\mathrm{~m}, 1 \mathrm{H}), 1.25(\mathrm{br}, 12 \mathrm{H})$. ${ }^{13} \mathrm{C}$ NMR (DMSO- $\left.d_{6}, 126 \mathrm{MHz}\right) \delta 176.9,160.3,156.8$, $155.3,152.1,148.1,147.5,147.4,145.8,143.9,137.4$, $127.6,123.9,122.1,121.0,117.3,116.9,115.7,112.0$, 107.1, 104.0, 101.2, 79.0, 76.3, 60.5, 56.1, 47.0, 46.4, 21.7, 20.4. $\mathrm{MS}\left(\mathrm{ESI}^{+}\right) \mathrm{m} / \mathrm{z}$ calcd for $\mathrm{C}_{32} \mathrm{H}_{34} \mathrm{NO}_{11} 608.21$, found $608.30(\mathrm{M}+\mathrm{H})^{+}$.

\section{3,5-dihydroxy-2-(3-(4-hydroxy-3-methoxyphenyl)-2-} (hydroxymethyl)-2,3-dihydrobenzo[b][1,4]dioxin-6-yl)-4oxo-4H-chromen-7-yl [1,4'-bipiperidine]-1'-carboxylate (3h)

Yellow solid (102.1 mg, purity $97.30 \%$, yield $30.3 \%$ ); mp $144-147^{\circ} \mathrm{C} ;{ }^{1} \mathrm{H}$ NMR (DMSO- $\left.d_{6}, 400 \mathrm{MHz}\right) \delta 12.45(\mathrm{~s}$, $1 \mathrm{H}), 9.90(\mathrm{~s}, 1 \mathrm{H}), 9.19(\mathrm{~s}, 1 \mathrm{H}), 7.84-7.82(\mathrm{~m}, 2 \mathrm{H}), 7.15(\mathrm{~d}$, $J=8.32,1 \mathrm{H}), 7.08(\mathrm{~d}, J=1.88,1 \mathrm{H}), 7.05(\mathrm{~d}, J=1.80$, $1 \mathrm{H}), 6.90(\mathrm{dd}, J=8.20,1.80,1 \mathrm{H}), 6.82(\mathrm{~d}, J=8.04,1 \mathrm{H})$, $6.61(\mathrm{~d}, J=1.96,1 \mathrm{H}), 5.03(\mathrm{t}, J=5.20,1 \mathrm{H}), 4.99(\mathrm{~d}, J=$ 7.92, 1H), 4.31-4.27 (m, 1H), 4.17 (br, 1H), 4.06 (br, 1H), $3.79(\mathrm{~s}, 3 \mathrm{H}), 3.59-3.56(\mathrm{~m}, 1 \mathrm{H}), 3.38-3.36(\mathrm{~m}, 3 \mathrm{H}), 3.05(\mathrm{t}$, $J=13.00,1 \mathrm{H}), 2.90(\mathrm{t}, J=12.80,1 \mathrm{H}), 2.49$ (br, 3H), 1.79-1.77 (m, 2H), 1.50-1.40 (m, 8H). ${ }^{13} \mathrm{C}$ NMR (DMSO$\left.d_{6}, 126 \mathrm{MHz}\right) \delta 176.9,160.3,156.8,155.2,152.0,148.0$, $147.5,147.4,145.8,143.9,137.5,127.6,123.9,122.0$, $121.0,117.3,116.8,115.7,112.0,107.2,104.3,101.5$, 79.0, 76.3, 61.7, 60.5, 56.1, 50.0, 44.4, 43.9, 27.9, 27.5, 26.3, 24.7. $\mathrm{MS}\left(\mathrm{ESI}^{+}\right) \mathrm{m} / \mathrm{z}$ calcd for $\mathrm{C}_{36} \mathrm{H}_{39} \mathrm{~N}_{2} \mathrm{O}_{11}$ 675.26, found $675.50(\mathrm{M}+\mathrm{H})^{+}$.

(2R,3R)-3,5-dihydroxy-2-(3-(4-hydroxy-3-methoxyphenyl)-2(hydroxymethyl)-2,3-dihydrobenzo[b][1,4]dioxin-6-yl)-4oxochroman-7-yl methoxy(methyl)carbamate (3i)

Yellow solid (37.2 mg, purity $97.80 \%$, yield $13.1 \%$ ); mp $114-117^{\circ} \mathrm{C} ;{ }^{1} \mathrm{H}$ NMR (DMSO- $\left.d_{6}, 400 \mathrm{MHz}\right) \delta 12.49$ (s, $1 \mathrm{H}$ ), 
$9.94(\mathrm{~s}, 1 \mathrm{H}), 9.22(\mathrm{~s}, 1 \mathrm{H}), 7.84-7.81(\mathrm{~m}, 2 \mathrm{H}), 7.15(\mathrm{~d}, J=$ $5.12,1 \mathrm{H}), 7.14(\mathrm{~d}, J=5.08,1 \mathrm{H}), 7.06(\mathrm{~d}, J=1.48,1 \mathrm{H}), 6.91$ $(\mathrm{dd}, J=8.12,1.52,1 \mathrm{H}), 6.83(\mathrm{~d}, J=8.04,1 \mathrm{H}), 6.67(\mathrm{~d}, J=$ $1.96,1 \mathrm{H}), 5.06(\mathrm{t}, J=5.16,1 \mathrm{H}), 4.98(\mathrm{~d}, J=7.92,1 \mathrm{H})$, 4.31-4.27 (m, 1H), $3.80(\mathrm{~s}, 3 \mathrm{H}), 3.75(\mathrm{~s}, 3 \mathrm{H}), 3.59-3.56(\mathrm{~m}$, 1H), 3.38-3.34 (m, 1H), $3.25(\mathrm{~s}, 3 \mathrm{H}) .{ }^{13} \mathrm{C}$ NMR (DMSO- $d_{6}$, $126 \mathrm{MHz}) \delta 176.9,160.4,156.0,155.2,153.3,148.0,147.5$, $147.5,145.8,143.9,137.6,127.6,123.8,122.0,121.0,117.3$, 116.9, 115.7, 112.0, 107.6, 104.3, 101.6, 79.0, 76.3, 61.7, 60.5, 56.1, 35.6. $\mathrm{MS}\left(\mathrm{ESI}^{+}\right) \mathrm{m} / \mathrm{z}$ calcd for $\mathrm{C}_{28} \mathrm{H}_{26} \mathrm{NO}_{12} 568.15$, found $568.30(\mathrm{M}+\mathrm{H})^{+}$.

\section{General procedure for the synthesis of compounds 4a-b}

To a solution of silibinin $(241.2 \mathrm{mg}, 0.50 \mathrm{mmol}, 1.0 \mathrm{eq})$ in DMF $(3.6 \mathrm{~mL}, 15.0 \mathrm{vol})$ was added $\mathrm{K}_{2} \mathrm{CO}_{3}$ (138.2 mg, $1.0 \mathrm{mmol}, 2.0 \mathrm{eq})$ and carbamyl chloride $\mathbf{1 a}-\mathbf{b}(1.03 \mathrm{mmol}$, $2.05 \mathrm{eq}$ ). The mixture was stirred at $25^{\circ} \mathrm{C}$ under $\mathrm{N}_{2}$ atmosphere for $12 \mathrm{~h}$. After completion of the reaction (monitored by TLC), the reaction mixture diluted with water $(20 \mathrm{~mL})$ and extracted with ethyl acetate $(20 \mathrm{~mL})$ twice. The combined organic layer was washed with brine $(20 \mathrm{~mL})$, dried over $\mathrm{Na}_{2} \mathrm{SO}_{4}$ and filtered. The filtrate was concentrated to give the crude product. The residue was purified by column chromatography $\left(\mathrm{CH}_{2} \mathrm{Cl}_{2} /\right.$ $\mathrm{MeOH}=15: 1)$ to give the title compound $\mathbf{4 a - b}$.

\section{5-hydroxy-2-(3-(4-hydroxy-3-methoxyphenyl)-2- (hydroxymethyl)-2,3-dihydrobenzo[b] [1,4]dioxin-6-yl)-4-oxo- 4H-chromene-3,7-diyl bis(morpholine-4-carboxylate) (4a)}

White solid (39.2 mg, purity $95.71 \%$, yield $11.1 \%$ ); mp $143-146{ }^{\circ} \mathrm{C} ;{ }^{1} \mathrm{H}$ NMR (DMSO- $\left.d_{6}, 400 \mathrm{MHz}\right) \delta 12.18(\mathrm{~s}$, $1 \mathrm{H}), 9.20(\mathrm{~s}, 1 \mathrm{H}), 7.55(\mathrm{~s}, 1 \mathrm{H}), 7.53(\mathrm{dd}, J=8.58,1.96$, $1 \mathrm{H}), 7.21(\mathrm{~d}, J=8.56,1 \mathrm{H}), 7.17(\mathrm{~d}, J=2.04,1 \mathrm{H}), 7.06(\mathrm{~d}$, $J=1.76,1 \mathrm{H}), 6.90(\mathrm{dd}, J=8.20,1.76,1 \mathrm{H}), 6.83(\mathrm{~d}, J=$ $8.08,1 \mathrm{H}), 6.75(\mathrm{~d}, J=2.04,1 \mathrm{H}), 5.05(\mathrm{t}, J=5.40,1 \mathrm{H})$, $5.01(\mathrm{~d}, J=7.92,1 \mathrm{H}), 4.35-4.31(\mathrm{~m}, 1 \mathrm{H}), 3.79(\mathrm{~s}, 3 \mathrm{H})$, $3.68(\mathrm{t}, J=4.32,8 \mathrm{H}), 3.60-3.57(\mathrm{~m}, 5 \mathrm{H}), 3.44-3.36(\mathrm{~m}$, $5 \mathrm{H}) .{ }^{13} \mathrm{C}$ NMR (DMSO- $\left.d_{6}, 126 \mathrm{MHz}\right) \delta 176.7,160.6$, $157.3,157.0,155.8,152.1,148.1,147.5,147.2,144.2$, $131.4,127.4,122.4,121.8,121.0,117.8,117.3,115.7$, $112.0,108.0,105.6,102.0,79.0,76.3,66.4,66.1,60.4$, 56.0, 45.4, 45.2, 44.7, 44.3. $\mathrm{MS}\left(\mathrm{ESI}^{+}\right) \mathrm{m} / \mathrm{z}$ calcd for $\mathrm{C}_{35} \mathrm{H}_{35} \mathrm{~N}_{2} \mathrm{O}_{14}$ 707.67, found $707.40(\mathrm{M}+\mathrm{H})^{+}$.

\section{5-hydroxy-2-(3-(4-hydroxy-3-methoxyphenyl)-2- (hydroxymethyl)-2,3-dihydrobenzo[b][1,4]dioxin-6-yl)-4- oxo-4H-chromene-3,7-diyl bis(dimethylcarbamate) (4b)}

White solid ( $86.2 \mathrm{mg}$, purity $92.26 \%$, yield $27.7 \%$ ); $\mathrm{mp}$ $135-138{ }^{\circ} \mathrm{C}$; ${ }^{1} \mathrm{H}$ NMR (DMSO- $\left.d_{6}, 400 \mathrm{MHz}\right) \delta 12.17(\mathrm{~s}$, $1 \mathrm{H}), 9.20$ (s, 1H), 7.55 (d, $J=8.58,1 \mathrm{H}), 7.52$ (dd, $J=8.56$,
$2.12,1 \mathrm{H}), 7.19(\mathrm{~d}, J=8.56,1 \mathrm{H}), 7.12(\mathrm{~d}, J=2.00,1 \mathrm{H}), 7.06$ $(\mathrm{d}, J=1.72,1 \mathrm{H}), 6.90(\mathrm{dd}, J=8.20,1.76,1 \mathrm{H}), 6.82(\mathrm{~d}, J=$ $8.00,1 \mathrm{H}), 6.70(\mathrm{~d}, J=2.04,1 \mathrm{H}), 5.04(\mathrm{t}, J=5.44,1 \mathrm{H}), 5.01$ $(\mathrm{d}, J=7.96,1 \mathrm{H}), 4.35-4.31(\mathrm{~m}, 1 \mathrm{H}), 3.78(\mathrm{~s}, 3 \mathrm{H}), 3.60-3.56$ (m, 1H), 3.42-3.35 (m, 1H), $3.10(\mathrm{~s}, 3 \mathrm{H}), 3.05(\mathrm{~s}, 3 \mathrm{H}), 2.93$ $(\mathrm{s}, 3 \mathrm{H}), 2.91(\mathrm{~s}, 3 \mathrm{H}) .{ }^{13} \mathrm{C}$ NMR (DMSO-d $\left.6,126 \mathrm{MHz}\right) \delta$ 176.8, 160.5, 157.5, 156.7, 155.8, 153.1, 152.9, 148.1, 147.5, 147.1, 144.2, 131.8, 127.4, 122.5, 121.9, 121.0, 117.8, 117.3, 115.7, 112.1, 107.8, 105.5, 101.9, 79.0, 76.3, 60.4, 56.1, 37.0, 36.8, 36.8, 36.6. $\mathrm{MS}\left(\mathrm{ESI}^{+}\right) \mathrm{m} / \mathrm{z}$ calcd for $\mathrm{C}_{31} \mathrm{H}_{31} \mathrm{~N}_{2} \mathrm{O}_{12}$ 623.59 , found $623.40(\mathrm{M}+\mathrm{H})^{+}$.

\section{CCK-8 assay}

In vitro cytotoxicity of the synthesized compounds, control drugs silybin and DHS was measured using the CCK-8 assay. In brief, a suspension of cells (around 5000 cells per well for MCF-7, NCI-H1299, HepG2 and HT29) was inoculated into 96 -well plates and incubated at $37^{\circ} \mathrm{C}$ in an atmosphere with $5 \% \mathrm{CO}_{2}$ for $24 \mathrm{~h}$. Then, solutions (PBS buffer containing $1 \%$ DMSO) of the prepared compounds and control drugs at a series of concentrations were added into the 96-well plates. After incubation for another $48 \mathrm{~h}$, $10 \mu \mathrm{L}$ of CCK-8 solution was added into each well and the plates were again incubated at $37^{\circ} \mathrm{C}$ for another $2 \mathrm{~h}$. The optical density (OD) values were measured using a microplate reader at $450 \mathrm{~nm}$ and expressed as $\mathrm{IC}_{50}$ values which was calculated with SPSS.

\section{Molecular docking}

The co-crystal structure of Hsp90 in complex with XL888 [37] (PDB code 4AWO) was obtained from the PDB (http://www.pdb.org) and used for the docking calculation in MOE (version 2020). 3D structures of the ligands were constructed, and their energy minimization were performed. For preparing the protein receptors, the hydrogen atoms were added into the X-ray structure and the Triangle Matcher-force field was employed. The site sphere was determined based on the binding location of XL888 in Hsp90. The ligand XL888 and water were removed from the binding site, and compound $\mathbf{2 f}, \mathbf{2 h}, \mathbf{3 e}, \mathbf{3 g}$ was docked into the prepared site in Hsp90, respectively. After evaluating the types of interactions between the tested compounds and Hsp90, the final binding conformation in Hsp90 was confirmed based on the calculated energy.

\section{Compliance with ethical standards}

Conflict of interest The authors declare no competing interests.

Publisher's note Springer Nature remains neutral with regard to jurisdictional claims in published maps and institutional affiliations. 


\section{References}

1. World Health Organization. Fact sheet detail. https://www.who. int/news-room/fact-sheets/detail/cancer. Accessed Dec 2021

2. Ali I, Lone MN, Al-Othman ZA, Al-Warthan A, Sanagi MM. Heterocyclic scaffolds: centrality in anticancer drug development. Curr Drug Targets. 2015;16:711-34. https://doi.org/10.2174/ 1389450116666150309115922

3. Sung H, Ferlay J, Siegel RL, Laversanne M, Soerjomataram I, Jemal A, et al. Global cancer statistics 2020: GLOBOCAN estimates of incidence and mortality worldwide for 36 cancers in 185 countries. CA Cancer J Clin. 2021;71:209-49. https://doi.org/10. 3322/caac. 21660

4. Cragg GM, Grothaus PG, Newman DJ. Impact of natural products on developing new anti-cancer agents. Chem Rev. 2009;109:3012-43. https://doi.org/10.1021/cr900019j

5. Ali I, Lone MN, Suhail M, Mukhtar SD, Asnin L. Advances in nanocarriers for anticancer drugs delivery. Curr Med Chem. 2016;23:2159-87. https://doi.org/10.2174/ 0929867323666160405111152

6. Kroll DJ, Shaw HS, Oberlies NH. Milk thistle nomenclature: why it matters in cancer research and pharmacokinetic studies. Integr Cancer Ther. 2007;6:110-9. https://doi.org/10.1177/1534735407301825

7. Gazak R, Walterova D, Kren V. Silybin and silymarin - new and emerging applications in medicine. Curr Med Chem. 2007;14:315-38. https://doi.org/10.2174/092986707779941159

8. Kvasnicka F, Biba B, Sevcik R, Voldrich M, Kratka J. Analysis of the active components of silymarin. J Chromatogr A. 2003;990:239-45. https://doi.org/10.1016/S0021-9673(02)01971-4

9. Gazák R, Svobodová A, Psotová J, Sedmera P, Prikrylová V, Walterová D, Kren V. Oxidised derivatives of silybin and their antiradical and antioxidant activity. Bioorg Med Chem. 2004;12:5677-87. https://doi.org/10.1016/j.bmc.2004.07.064

10. Agarwal R, Agarwal C, Ichikawa H, Singh RP, Aggarwal BB. Anticancer potential of silymarin: from bench to bed side. Anticancer Res. 2006;26:4457-98. https://doi.org/10.1016/j. jembe.2004.06.008

11. Huber A, Thongphasuk P, Erben G, Lehmann WD, Tuma S, Stremmel W, Chamulitrat W. Significantly greater antioxidant anticancer activities of 2,3-dehydrosilybin than silybin. Biochim Biophys Acta. 2008;1780:837-47. https://doi.org/10.1016/j.bba gen.2007.12.012

12. Dzubak P, Hajduch M, Gazak R, Svobodova A, Psotova J, Walterova D, et al. New derivatives of silybin and 2,3-dehydrosilybin and their cytotoxic and P-glycoprotein modulatory activity. Bioorg Med Chem. 2006;14:3793-810. https://doi.org/ 10.1016/j.bmc.2006.01.035

13. Thongphasuk P, Stremmel W, Chamulitrat W. Potent direct or TNF-alpha-promoted anticancer effects of 2,3-dehydrosilybin: comparison study with silybin. Chemotherapy. 2008;54:23-30. https://doi.org/10.1159/000112314

14. Singh RP, Agarwal R. Prostate cancer prevention by silibinin. Curr Cancer Drug Targets. 2004;4:1-11. https://doi.org/10.2174/ 1568009043481605

15. Saller R, Brignoli R, Melzer J, Meier R. An updated systematic review with meta-analysis for the clinical evidence of silymarin. Forsch Komplementmed. 2008;15:9-20. https://doi.org/10.1159/ 000113648

16. Romanucci V, Agarwal C, Agarwal R, Pannecouque C, Iuliano M, Tommaso GD, et al. Silibinin phosphodiester glyco-conjugates: synthesis, redox behaviour and biological investigations. Bioorg Chem. 2018;77:349-59. https://doi.org/10.1016/j.bioorg.2018.01.026

17. Cufi S, Bonavia R, Vazquez-Martin A, Corominas-Faja B, Oliveras-Ferraros C, Cuyas E, et al. Silibinin meglumine, a watersoluble form of milk thistle silymarin, is an orally active anti- cancer agent that impedes the epithelial-to-mesenchymal transition (EMT) in EGFR-mutant non-small-cell lung carcinoma cells. Food Chem Toxicol. 2013;60:360-8. https://doi.org/10.1016/j.fct. 2013.07.063

18. Bijak M. Silybin, a major bioactive component of milk thistle (Silybum marianum L. Gaernt.)-chemistry, bioavailability, and metabolism. Molecules. 2017;22:1942-69. https://doi.org/10. 3390/molecules22111942

19. Hawke RL, Schrieber SJ, Soule TA, Wen ZM, Smith PC, Reddy $\mathrm{KR}$, et al. Silymarin ascending multiple oral dosing phase I study in noncirrhotic patients with chronic hepatitis c. J Clin Pharmacol. 2010;50:434-49. https://doi.org/10.1177/0091270009347475

20. Yu JN, Zhu Y, Wang L, Peng M, Tong SS, Cao X, et al. Enhancement of oral bioavailability of the poorly water-soluble drug silybin by sodium cholate/phospholipid-mixed micelles. Acta Pharmacol Sin. 2010;31:759-64. https://doi.org/10.1038/aps. 2010.55

21. Yang LX, Huang KX, Li HB, Gong JX, Wang F, Feng YB, et al. Design, synthesis, and examination of neuron protective properties of alkenylated and amidated dehydro-silybin derivatives. J Med Chem. 2009;52:7732. https://doi.org/10.1021/ jm900735p

22. Ray S, Chaturvedi D. Application of organic carbamates in drug design. Part 1: anticancer agents - recent reports. Drugs Future. 2004;29:343-57. https://doi.org/10.1358/dof.2004.029.04.787236.

23. Rahmanthullan SM, Tidwell RR, Jones SK, Hall JE, Boykin DW. Carbamate prodrugs of $\mathrm{N}$-alkylfuramidines. Eur J Med Chem. 2008;43:174-7. https://doi.org/10.1016/j.ejmech.2007.03.009

24. Ghosh AK, Brindisi M. Organic carbamates in drug design and medicinal chemistry. J Med Chem. 2015;58:2895-40. https://doi. org/10.1021/jm501371s

25. Greenwald RB, Pendri A, Bolikal D. Highly water soluble taxol derivatives: 7-polyethylene glycol carbamates and carbonates. J Org Chem. 1995;60:331-6. https://doi.org/10.1021/jo00107a010

26. Azzolini M, Mattarei A, La Spina M, Marotta E, Zoratti M, Paradisi C, et al. Synthesis and evaluation as prodrugs of hydrophilic carbamate ester analogues of resveratrol. Mol Pharm. 2015;12:3441-54. https://doi.org/10.1021/acs.molpharma ceut. 5 b00464

27. Cheong JE, Zaffagni M, Chung I, Xu YJ, Wang YQ, Jernigan FE, et al. Synthesis and anticancer activity of novel water soluble benzimidazole carbamates. Eur J Med Chem. 2018;144:372-85. https://doi.org/10.1016/j.ejmech.2017.11.037

28. Takaoka K, Tatsu Y, Yumoto N, Nakajima T, Shimamoto K. Synthesis of carbamate-type caged derivatives of a novel glutamate transporter blocker. Bioorg Med Chem. 2004;12:3687-94. https://doi.org/10.1016/j.bmc.2004.04.011

29. Borrel C, Thoret S, Cachet X, Guenard D, Tillequin F, Koch M, et al. New antitubulin derivatives in the combretastatin A4 series: synthesis and biological evaluation. Bioorg Med Chem. 2005;13:3853-64. https://doi.org/10.1016/j.bmc.2005.02.039

30. Holas T, Vavrova K, Sima M, Klimentova J, Hrabalek A. Synthesis and transdermal permeation-enhancing activity of carbonate and carbamate analogs of Transkarbam 12. Bioorg Med Chem. 2006;14:7671-80. https://doi.org/10.1016/j.bmc. 2006.08.014

31. Zhao H, Brandt GE, Galam L, Matts RL, Blagg BSJ. Identification and initial SAR of silybin: An Hsp90 inhibitor. Bioorg Med Chem Lett. 2011;21:2659-64. https://doi.org/10.1016/j.bmcl. 2010.12.088

32. Riebold M, Kozany C, Freiburger L, Sattler M, Buchfelder M, Hausch F, et al. A C-terminal HSP90 inhibitor restores glucocorticoid sensitivity and relieves a mouse allograft model of Cushing disease. Nat Med. 2015;21:276-80. https://doi.org/10. 1038/nm.3776 
33. Tiwari P, Kumar A, Balakrishnan S, Kushwaha HS, Mishra KP. Silibinin-induced apoptosis in MCF7 and T47D human breast carcinoma cells involves caspase- 8 activation and mitochondrial pathway. Cancer Investig. 2011;29:12-20. https://doi.org/10. 3109/07357907.2010.535053

34. Kim TH, Woo JS, Kim YK, Kim KH. Silibinin induces cell death through reactive oxygen species-dependent downregulation of notch1/ERK/Akt signaling in human breast cancer cells. J Pharmacol Exp Ther. 2014;349:268-78. https://doi.org/10.1124/jpet.113.207563

35. Kil WH, Kim SM, Lee JE, Park KS, Nam SJ. Anticancer effect of silibinin on the xenograft model using MDA-MB-468 breast cancer cells. Ann Surg Treat Res. 2014;87:167-73. https://doi.org/ 10.4174/astr.2014.87.4.167

36. Xu P, Yin Q, Shen J, Chen LL, Yu HJ, Zhang ZW, et al. Synergistic inhibition of breast cancer metastasis by silibinin-loaded lipid nanoparticles containing TPGS. Int J Pharm. 2013;454:21-30. https://doi.org/10.1016/j.ijpharm. 2013.06.053

37. Bussenius J, Blazey CM, Aay N, Anand NK, Arcalas A, Baik TG, et al. Discovery of XL888: a novel tropane-derived small molecule inhibitor of HSP90. Bioorg Med Chem Lett. 2012;22:5396-404. https://doi.org/10.1016/j.bmcl.2012.07.052 